\title{
6. ANGULAR MOMENTA OF LATE-TYPE SPIRAL GALAXIES
}

\author{
N. HEIDMANN \\ Observatoire de Meudon, Meudon, France
}

\begin{abstract}
The angular momenta of galaxies may be evaluated from their photometry. The mass to light ratio in NGC 224 is discussed and the calculation is applied to two late-type spirals.
\end{abstract}

\section{Introduction}

In a previous study an attempt was made towards the investigation of specific angular momenta of spirals by the use of indicative specific angular momenta defined by

$$
A_{\mathrm{i}}=0.10 a V_{\mathrm{m}}
$$

where $A_{\mathrm{i}}$ is in kpc km s${ }^{-1}, a$ is the $26.5 m_{\mathrm{pg}}$ per square arcsec photometric diameter in $\mathrm{kpc}$ and $V_{\mathrm{m}}$ the maximum rotational velocity in $\mathrm{km} \mathrm{sec}^{-1}$ (Heidmann, 1969). The largest $A_{\mathrm{i}}$ values are obtained mostly for the Sbc type, suggesting that there may be a relation between the angular momentum and the degree of development of spiral arms.

However other factors may be of importance and the relation between indicative specific angular momentum $A_{\mathrm{i}}$ and real specific angular momentum $A$ has first to be closely investigated.

The evaluation of the angular momentum of a galaxy from the rotation curve is very sensitive to its shape after the turnover point. If $r_{\mathrm{m}}$ is the turnover radius and $V_{\mathrm{m}}$ the maximum rotational velocity, the specific angular momentum for a flat disk is:

$$
A=k r_{\mathrm{m}} V_{\mathrm{m}}
$$

where $k$ is a coefficient dependent on the shape of the rotation curve $V(r)$. For example, Toomre's (1963) calculations show that in two cases in which $V\left(2 r_{\mathrm{m}}\right)=0.9 V_{\mathrm{m}}$ or $0.7 V_{\mathrm{m}}, k$ differs by a factor 4 . Similar results are obtained from Takase and Kinoshita's (1967) calculations.

As it is difficult to measure radial velocities after the turnover radius, we attempt to derive angular momenta from photometric profiles. With values of the mass to luminosity ratio, the brightness distribution gives the density distribution, which in turn gives the potential, then the force, the velocity and the angular momentum.

With respect to the axis of cylindrical coordinates $(r, \theta, z)$, the specific angular momentum of a galaxy is:

$$
A=\frac{1}{M_{\mathrm{T}}} \sum_{\text {particles }} r \Theta m,
$$

where $M_{\Upsilon}$ is the total mass, $\Theta$ the tangential velocity and $m$ the mass of a particle. 
A first summation on the particles in a small volume element changes $\Theta$ into $V_{0}$, the centroid velocity. For a stationary system with rotational symmetry, Jeans' (1922) equations may be used. The calculation is quite easy in case the galaxy is flat enough to assume that:

(i) the velocity dispersions are negligeable,

(ii) the circular velocity $V_{\mathrm{c}}(r, z)$ is nearly independent of $z$ inside the system.

If the system has an equatorial plane of symmetry, the momentum is then:

$$
A=\frac{4 \pi}{M_{\mathrm{T}}} \int_{0}^{\infty} r^{5 / 2}\left[K_{\mathrm{r}}(r, 0)\right]^{1 / 2} \int_{0}^{\infty} \varrho(r, z) \mathrm{d} z \mathrm{~d} r,
$$

where $K_{\mathrm{r}}(r, 0)$ is the radial force in the equatorial plane and $\varrho$ the density. Usually the main contribution to $A$ will arise from intermediate $r$ values, so that the two above assumptions have to be fulfilled especially for these $r$ values. Formula (4) will be applied to late type spirals.

\section{Photometric Profiles}

De Vaucouleurs (1962) has shown that the photometric profile of elliptical galaxies follows a ' $r^{1 / 4}$, law and that the profile of late-type spirals follows an exponential law. He has also shown (1958) that the profile of an intermediate type spiral such as NGC 224 can be represented by the sum of a ' $r^{1 / 4}$ ' profile due to the bulge (spheroidal component) and an exponential profile due to the disk (flat component). We may assume that in a first approximation the profile of a galaxy may be represented by the sum of two such profiles.

\section{Mass to Luminosity Ratio}

The global mass to luminosity ratio for ellipticals is 20 (Holmberg, 1964) and for late type spirals it is 4 (Heidmann, 1969); here the luminosities are corrected for absorption according to Holmberg (1964).

The mass to luminosity ratio $f(r)$ at various distances $r$ from the center of a galaxy has been worked out for the Large Magellanic Cloud by De Vaucouleurs (1960) using two Perek spheroids. It is constant for $r>2^{\circ}$; for $r<2^{\circ}$ it is smaller and decreases by two thirds for $r=0$ according to this method; however, in this analysis, there was no rotation observation available for $r<1.5^{\circ}$. It seems that for this galaxy, made up practically of a flat component, $f(r)$ can be taken to be a constant in most of the object.

The value of $f(r)$ has also been given by Gottesman et al. (1966) for NGC 224 using a Brandt curve (Brandt and Scheer, 1965) with index $\frac{3}{2}$ to fit their $21-\mathrm{cm}$ observations. It is reproduced in Figure 1. In a dynamical study of the central parts of NGC 224 up to $r=10^{\prime}$, Kinman (1965) obtained $f \sim 50$. When comparing his rotation curves with the one observed by Lallemand et al. (1960), we obtain $f=25$. These values are plotted in Figure 1. 
Though the quasistellar nucleus of NGC 224 might play a special role, Kinman's values suggest that $f$ is indeed large in the central parts of NGC 224, as for elliptical galaxies. It is possible that the $f(r)$ values of Gottesman et al. in the central regions are smaller because Brandt's formalism is not valid for a mass distribution with large axis ratios and the radiotelescope beam has the effect to lower the slope of the rotation curve.

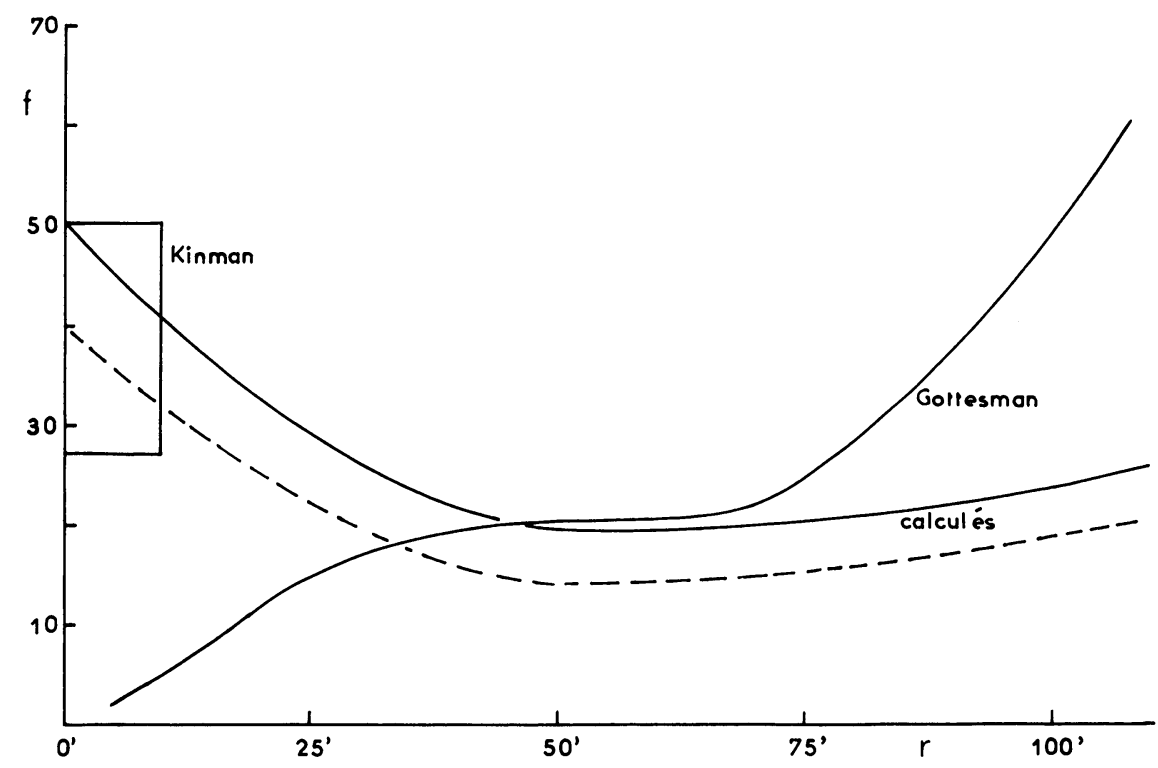

Fig. 1. Mass to luminosity ratio $f$, in solar units, vs. the distance from the center $r$ in arc min for NGC 224. The values from Gottesman $e t$ al. and from Kinman are plotted. The lines calculés are calculated for $f_{\mathrm{s}}=50, f_{\mathrm{p}}=15$ (full line) and for $f_{\mathrm{s}}=40, f_{\mathrm{p}}=10$ (broken line).

Using the two profiles given by De Vaucouleurs (1958) for the spheroidal and for the flat components of NGC 224 with the $f$ values $f_{\mathrm{s}}=50$ and $f_{\mathrm{f}}=15$ for each component respectively, we obtain the $f(r)$ full line curve labelled calculés in Figure 1. Note that the $f$ values here are not corrected for absorption. For a total absorption of 1 magnitude, assumed to be the same for the bulge and for the disk (cf. De Vaucouleurs 1958 for discussion), the corrected values would be 20 and 6 . The broken line is for $f_{\mathrm{s}}=40$ and $f_{\mathrm{f}}=10$.

The agreement with Kinman's values for small $r$ and with Gottesman's values for intermediate $r$ is satisfactory. For large $r$, Gottesman's values are larger by about a factor 2. This may be due to an overestimation of the surface density as it appears from comparison with the two best solutions which Roberts (1966) obtained from his observations. Figure 2 is a plot of the surface density $\sigma(r)$ and shows that Roberts' values are 2 or 4 times smaller than Gottesman's in the region 70-110' (and are larger for small $r$ values). This difference may be due to beamwidth and to bandwidth effects and to the fact that Gottesman et al. use mean velocities while Roberts uses corrected 
peak velocities which are closer to rotational velocities. Roberts' values for a Brandt curve with index $\frac{3}{2}$ are in agreement with the $f(r)$ curve calculated with the hypothesis of constant values for $f_{\mathrm{s}}$ and $f_{\mathrm{f}}$.

It may then be assumed that the mass to luminosity ratio $f$ of each of the spheroidal and flat components of a galaxy is the same at all point of each component.

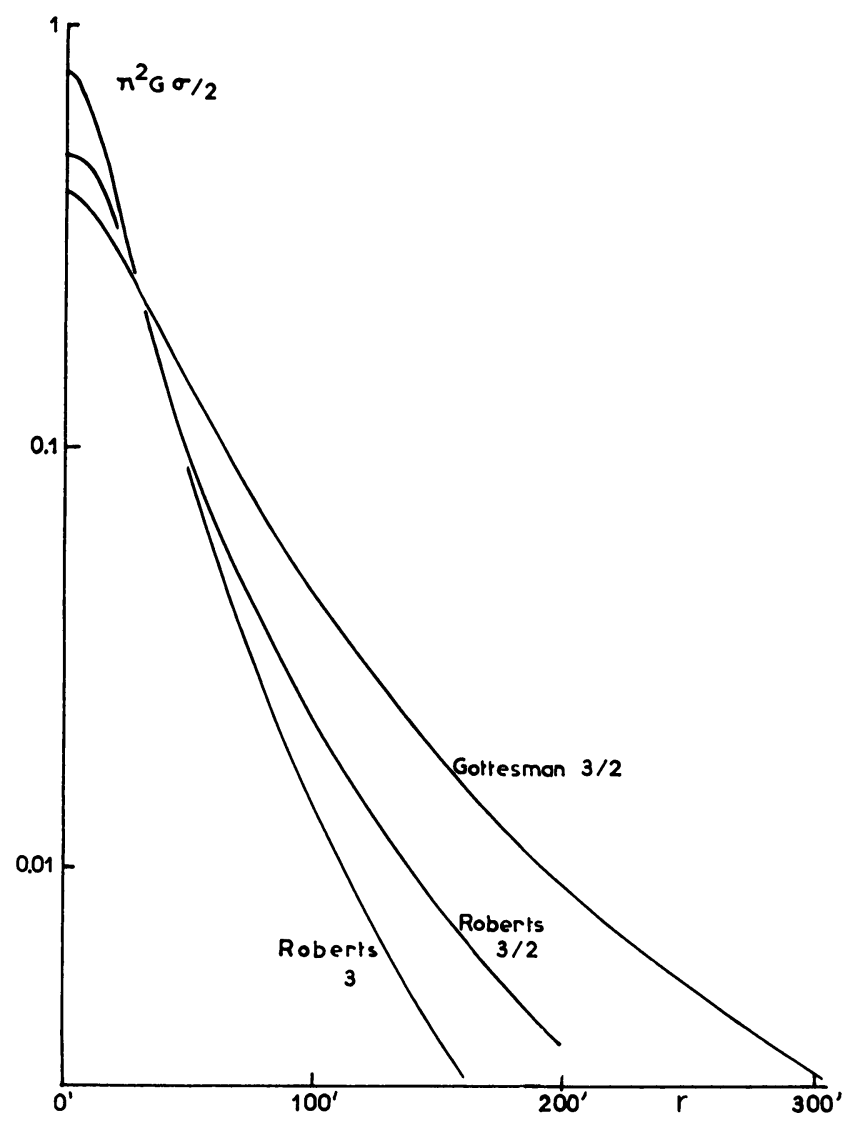

Fig. 2. Surface density $\sigma$ times $\pi^{2} G / 2$, in $10^{7} \mathrm{cgs}$ units, for NGC 224 vs. the distance from the center $r$ in arcmin. The curves are from Gottesman et al. and from Roberts with the indicated Brandt indices.

\section{Momentum of Late Type Spirals}

The specific angular momentum of late type spirals is the easiest to evaluate by the photometric method. There is only a flat component and Equation (4) may be used. Integration along $z$ introduces the surface density, which is exponential since $f$ is constant inside the component and the brightness is exponential. The calculation of $K_{\mathrm{r}}$ has been explicitly made in this case by Wyse and Mayall (1942). Using it, the 
specific angular momentum in $\mathrm{kpc} \mathrm{km} \mathrm{s}^{-1}$ is:

$$
A=0.325\left(D^{3} R^{3} f(b / a) I_{0}\right)^{1 / 2}
$$

where $D$ is the distance in $\mathrm{Mpc}, f$ the mass to luminosity ratio in solar units corrected for absorption, $b / a$ the axis ratio, $I_{0}$ the central brightness corrected for absorption in units of 25 magnitude per square arcsec and $R$ the distance in arcmin at which the brightness has fallen to the value $I_{0} e^{-4}$.

This calculation is applied to two late type spirals for which photometric profiles follow the exponential law: NGC 300 and LMC. The profiles are corrected for absorption in our Galaxy $\left(\Delta m_{\mathrm{g}}\right)$ and for internal absorption $\left(\Delta m_{\mathrm{i}}\right)$ according to Holmberg (1964); $B$ magnitudes are reduced to the $p g$ system by subtracting 0.1 . Values and references are given in Table $\mathrm{I}$.

\section{TABLE I}

Data for galaxies for which the angular momentum $A$ is evaluated from the photometry

\begin{tabular}{lccl} 
& NGC 300 & LMC & Units \\
\cline { 3 - 4 } & & & \\
type & Sd (d) & Sm (d) & \\
$I_{0}$ obs. & $21.7(\mathrm{a})$ & $21.5(\mathrm{~b})$ & $m_{p g \operatorname{arcsec}^{-2}}$ \\
$R$ & $13(\mathrm{a})$ & $370(\mathrm{~b})$ & $\operatorname{arcmin}$ \\
$\Delta m_{\mathrm{g}}$ & $0.2(\mathrm{a})$ & $0.4(\mathrm{~b})$ & $m_{p \mathrm{~g}}$ \\
$\Delta m_{\mathrm{i}}$ & $0.3(\mathrm{c})$ & $0.3(\mathrm{c})$ & $m_{p g}$ \\
$I_{0}$ corr. & 33 & 48 & $25 m_{p \mathrm{~g} \mathrm{arcsec}}^{-2}$ \\
$b / a$ & $0.70(\mathrm{~d})$ & $0.89(\mathrm{~b})$ & \\
$D$ & $2.5(\mathrm{e})$ & $0.048(\mathrm{f})$ & $\mathrm{Mpc}$ \\
$A$ & 580 & 320 & $\mathrm{kpc} \mathrm{km} \mathrm{s}^{-1}$ \\
$A_{\mathrm{i}}$ & 340 & 170 & $\mathrm{kp} \mathrm{km} \mathrm{s}^{-1}$
\end{tabular}

(a) G. de Vaucouleurs and Page (1962); (b) G. de Vaucouleurs (1960);

(c) Holmberg (1964); (d) G. and A. de Vaucouleurs (1964); (e) Bottinelli et al. (1968); (f) Sandage (1962).

We hope to extend to more late type and to other type galaxies the calculation of momenta using the photometric data. The case of the intermediate types Sc-Scd might be difficult to handle because a small spheroidal component may exist which barely affects the luminosity profile but which may give an important contribution to the density. For example, NGC 598 has a small spheroidal component with diameter about 4' (De Vaucouleurs, private communication) of which the photometry has not yet been done and which is probably responsible of the parabolic shape of the central part of the rotation curve obtained by Courtès' group (Baudel, private communication). The spatial flatness of the galaxy allows use of Equation (4) but the decomposition into two components may be difficult.

For comparison, the indicative specific angular momenta $A_{\mathrm{i}}$ are also listed in the Table I. A further comparison for an earlier type spiral is obtained from data for our 
Galaxy. Integration of the momentum distributions of Innanen's (1966) models yields $A=900-1300 \mathrm{kpc} \mathrm{km} \mathrm{s}{ }^{-1}$; Kinman's (1959) study of globular clusters gives $A=$ $800 \mathrm{kpc} \mathrm{km} \mathrm{s}^{-1}$. To obtain the indicative specific momentum the photometric diameter of our Galaxy may be estimated from the neutral hydrogen diameter through a statistical relation found by Bottinelli (private communication) between type and hydrogen diameter to photometric diameter ratio: $a \sim 40 \mathrm{kpc}$. With $V_{\mathrm{m}}=260 \mathrm{~km} \mathrm{~s}^{-1}$ (Kerr, private communication), the indicative momentum is $A_{\mathrm{i}} \sim 1040 \mathrm{kpc} \mathrm{km} \mathrm{s}{ }^{-1}$, which is also close to $A$.

\section{Acknowledgements}

It is a pleasure to thank Drs. J. Heidmann and G. de Vaucouleurs for advice and discussions.

\section{References}

Bottinelli, L., Gouguenheim, L., Heidmann, J., and Heidmann, N.: 1968, Ann. Astrophys. 31, 205. Brandt, J. C. and Scheer, L. S.: 1965, Astron. J. 70, 471.

Gottesman, S. T., Davies, R. D., and Reddish, V. C.: 1966, Monthly Notices Roy. Astron. Soc. 133, 359.

Heidmann, N.: 1969, Astrophys. Letters 3, 153.

Holmberg, E.: 1964, Ark. Astron. 3, 387.

Innanen, K. A.: 1966, Astrophys. J. 143, 153.

Jeans, J. H.: 1922, Monthly Notices Roy. Astron. Soc. 82, 122.

Kinman, T. D.: 1959, Monthly Notices Roy. Astron. Soc. 119, 559.

Kinman, T. D.: 1965, Astrophys. J. 142, 1376.

Lallemand, A., Duchesne, M., and Walker, M. F.: 1960, Publ. Astron. Soc. Pacific 72, 76.

Roberts, M. S.: 1966, Astrophys. J. 144, 639.

Sandage, A.: 1962, IAU Symposium No. 15, p. 359.

Takase, B. and Kinoshita, H.: 1967, Publ. Astron. Soc. Japan 19, 409.

Toomre, A.: 1963, Astrophys. J. 138, 385.

Vaucouleurs, G. de: 1958, Astrophys. J. 128, 465.

Vaucouleurs, G. de: 1960, Astrophys. J. 131, 265.

Vaucouleurs, G. de: 1962, IAU Symposium No. 15, p. 3.

Vaucouleurs, G. de and Page, J.: 1962, Astrophys. J. 136, 107.

Vaucouleurs, G. and A. de: 1964, Reference Catalogue of Bright Galaxies, Univ. Texas Press, Austin. Wyse, A. B. and Mayall, N. U.: 1942, Astrophys. J. 95, 24. 